\title{
Stimulation of Propionyl CoA and $\beta$ - Methylcrotonyl CoA Carboxylase Activities in Human Leukocytes and Cultured Fibroblasts by Biotin
}

\author{
BARRY WOLF ${ }^{(28)}$ AND LEON E. ROSENBERG ${ }^{(30)}$ \\ Departments of Human Genetics and Pediatrics, Yale University School of Medicine, New Haven, Connecticut USA
}

\begin{abstract}
Summary
Initially, it was observed that whereas the propionyl CoA carboxylase (PCC) activity in peripheral blood leukocytes of a 13 . month-old girl with isolated PCC deficiency who had been given biotin supplements ( $4.5 \mathrm{mg} /$ day) for 6 months was only $3 \%$ of control, her $\beta$ MCC activity was greatly elevated at 20 times control activity. After biotin supplements were discontinued, $\beta M C C$ activity in leukocyte extracts declined over 3 wk to values just above the normal range with no associated change in PCC activity; readministration of biotin caused her $\beta$ MCC activity to rise in 3 days to the initial elevated values. These results prompted measurement of $\mathrm{PCC}$ and $\beta \mathrm{MCC}$ activities in leukocyte extracts of three normal adults given $20 \mathrm{mg}$ biotin orally per day. Their PCC and $\beta M C C$ activities increased 2- to 3-fold and 4- to 9-fold, respectively, within 7-10 days. After biotin was discontinued, both enzyme activities returned to normal in 4-5 wk. The effect of high concentrations of biotin and $\beta M C C$ activities was also investigated in cultured fibroblasts from three control subjects. PCC activity increased only $11 \%$ in normal fibroblasts grown in medium containing fetal bovine serum and high concentrations of biotin $(900$ $\mu \mathrm{g} / \mathrm{ml}$ ). However, PCC and $\beta M C C$ activities were increased 70 $75 \%$ and $120-140 \%$, respectively, when confluent normal fibroblasts were incubated in medium containing $10 \%$ human serum and $0.1-1.0 \mu \mathrm{g} / \mathrm{ml}$ biotin. This stimulation was independent of the fibroblast growth cycle. Furthermore, cycloheximide failed to inhibit the increase in PCC activity caused by biotin, suggesting that the enhanced activity in fibroblasts was not due to de novo carboxylase synthesis. These studies demonstrate that high concentrations of biotin can stimulate biotin-dependent carboxylases in human tissues, possibly by converting apocarboxylase to holocarboxylase or by activating preexisting inactive holocarboxylase.
\end{abstract}

\section{Speculation}

The stimulation of human propionyl $\operatorname{CoA}$ and $\beta$-methylcrotonyl CoA carboxylase activities in peripheral blood leukocytes in vivo and in cultured skin fibroblasts by high concentrations of biotin represents a promising model for investigating the cellular metabolism of biotin in man.

Propionic acidemia is a disorder of organic acid metabolism caused by a deficiency of propionyl COA carboxylase (PCC; EC 6.4.1.3) activity. PCC requires the covalently bound coenzyme, biotin, to sequentially aceept and transfer a carboxyl group from bicarbonate to propionyl CoA, forming D-methylmalonyl CoA (17). Biotin has, therefore, been suggested as a possible mode of therapy for patients with PCC deficiency (20).

Only three reported patients with propionic acidemia, however have been shown to be biotin-responsive $(2,9,23)$. These patients demonstrated marked clinical improvement, decreased serum pro- pionate, and decreased urinary propionate metabolites after biotin therapy. Deficiencies of two closely related biotin dependent enzymes, PCC and $\beta$-methylcrotonyl $\mathrm{CoA}$ carboxylase $(\beta \mathrm{MCC}$; EC 6.4.1.4), were demonstrated in fibroblast extracts from one of these patients (23). Both carboxylase activities returned to normal when these fibroblasts were grown in biotin-supplemented medium $(3,23)$. PCC deficiency was shown in fibroblasts from a second patient, but $\beta \mathrm{MCC}$ activity before and after biotin administration was not reported (2). The third patient had isolated PCC deficiency with normal $\beta$ MCC activity in peripheral blood leukocytes and in fibroblast extracts (9). When biotin was added to the fibroblast growth medium, an increase in PCC activity towards the normal range was observed. Although the PCC activity of isolated peripheral blood leukocytes was also stimulated after biotin administration, the corresponding $\beta \mathrm{MCC}$ activity was not reported.

To evaluate the effect of biotin on the carboxylase activities in vivo in another patient with isolated PCC deficiency (normal fibroblast $\beta \mathrm{MCC}$ activity), the present study was initiated. PCC and $\beta \mathrm{MCC}$ activities in extracts of peripheral blood leukocytes from a patient receiving high doses of oral biotin were compared to the activities in extracts of leukocytes after biotin was discontinued. Although her leukocyte extracts showed no increase in PCC activity while receiving biotin, her leukocyte $\beta$ MCC activity was greatly elevated above the normal range. This observation prompted further investigation of the effect of biotin on PCC and $\beta M C C$ activities in peripheral blood leukocytes from normal individuals receiving biotin, and in normal human fibroblasts cultured in biotin-supplemented medium.

\section{MATERIALS AND METHODS}

\section{SUBJECTS}

Patient K.K. was a 13-month-old severely developmentally delayed white female with PCC deficiency diagnosed at 6 months of age. PCC activity in her cultured fibroblast extracts was $3 \%$ of control; $\beta \mathrm{MCC}$ activity was normal. She had been given oral biotin supplements $(4.5 \mathrm{mg} /$ day) for 7 months before our investigation. Four normal adults, two males and two females, ranging in age from $24-44 \mathrm{yr}$, were also studied. Three of the controls (two males and one female) received $10 \mathrm{mg}$ of oral D-biotin (Sigma) twice a day for 13 days. The second female did not receive the biotin supplement and served as an untreated control. The controls were allowed unrestricted diets and no adjustments were made for low dietary biotin intake (10). Signed informed consent was obtained from the parents of K.K. and from the four adults.

\section{PERIPHERAL BLOOD LEUKOCYTE STUDIES}

PCC and $\beta$ MCC activities were measured in freshly isolated peripheral blood leukocytes as described (11, 24, 25). Enzyme 
activities were determined in leukocytes from the four controls as well as from the patient with PCC deficiency every 2-3 days during the course of this study. Enzyme activities are expressed as pmole of ${ }^{14} \mathrm{CO}_{2}$ fixed per min per $\mathrm{mg}$ extract protein. Protein was determined by a modification of the method of Lowry et al. (15). Total white blood cell and differential counts were obtained at regular intervals during the investigation.

\section{SKIN FIBROBLAST STUDIES}

Skin fibroblast cultures derived from two normal adult individuals (not participants in the leukocyte study) and from one normal child were grown in minimal essential medium (MEM) with glutamine $(2 \mathrm{mM})$, kanamycin $(100 \mu \mathrm{g} / \mathrm{ml})$ and either $10 \%$ fetal bovine serum (FBS) or 10\% human serum (HS). The biotin concentration of basal MEM with $10 \% \mathrm{FBS}$ or HS is $20 \mathrm{ng} / \mathrm{ml}$ (23). Fibroblasts were plated at near confluence $\left(5 \times 10^{5}\right.$ cells $/ 60$ $\mathrm{mm}$ Falcon tissue culture dish). After $24 \mathrm{hr}$, cells were washed with phosphate buffered saline, $\mathrm{pH}$ 7.4. Biotin, which had been incubated with serum for $30 \mathrm{~min}$, was then added to the culture medium at concentrations of $0.01-900 \mu \mathrm{g} / \mathrm{ml}$ as indicated. Biotin added directly to the medium gave similar results. Cycloheximide (Boehringer-Mannheim), was used to inhibit protein synthesis in fibroblast cultures under conditions previously described (26); protein synthesis was inhibited by more than $95 \%$ after $1 \mathrm{hr}$ incubation in cycloheximide $(15 \mu \mathrm{g} / \mathrm{ml})$. PCC and $\beta \mathrm{MCC}$ activities in fibroblast extracts were assayed as in leukocytes. Glutamate dehydrogenase (GDH) was measured as described by Beaufay et al. (4). Trypan blue was used to assess cell viability (18).

\section{RESULTS}

\section{HUMAN PERIPHERAL LEUKOCYTE STUDIES}

As shown in Figure 1, patient K.K.'s PCC activity in leukocyte extracts, while she received biotin $(4.5 \mathrm{mg} /$ day $)$ was $5 \%$ of mean normal activity, but simultaneously measured $\beta \mathrm{MCC}$ activity was
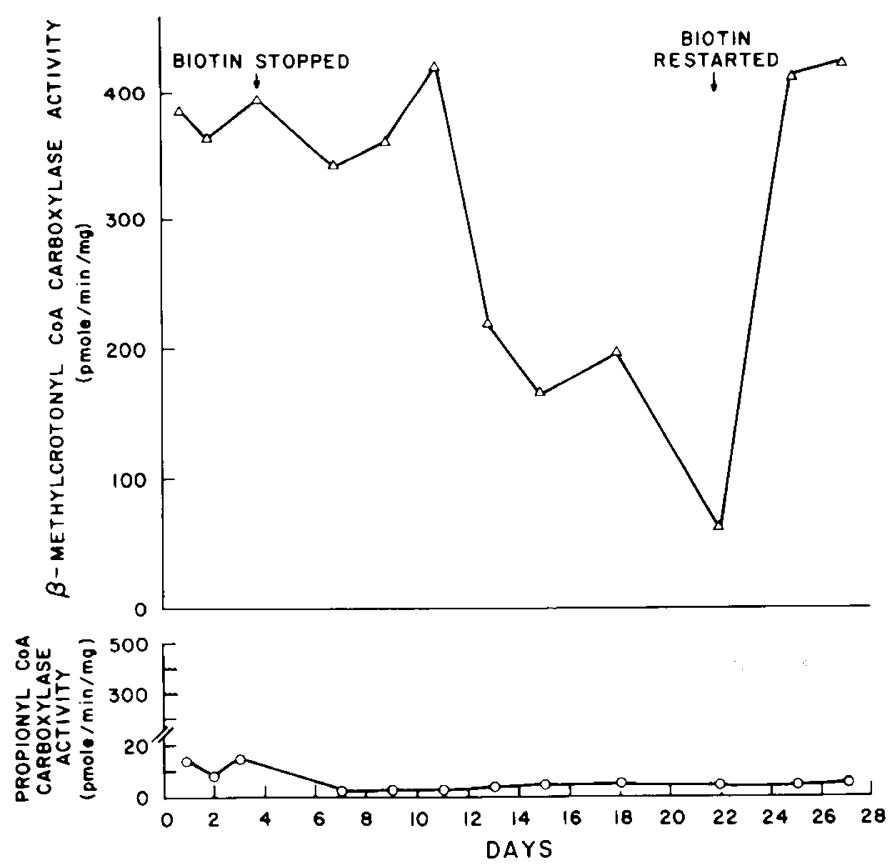

Fig. 1. $\beta \mathrm{MCC}(\triangle)$ and PCC $(O)$ activities in extracts of peripheral blood leukocytes obtained from a patient with PCC deficiency. Activities were measured while the patient received biotin, after biotin was discontinued (arrow, day 4), and after the readministration of biotin (arrow, day 22). The shaded areas represent the normal ranges for $\beta \mathrm{MCC}$ and PCC activities. over 20 times normal. $\beta \mathrm{MCC}$ activity in leukocyte extracts remained elevated for 1 wk after the biotin was discontinued, then slowly decreased over the next 11 days to $50 \mathrm{pmole} / \mathrm{min} / \mathrm{mg}$, a value just above the upper limit of normal. Biotin was then restarted at the same dose and, within 3 days, leukocyte $\beta \mathrm{MCC}$ activity returned to the initial elevated values. PCC activity remained essentially unchanged throughout the entire course of the study.

The PCC and $\beta \mathrm{MCC}$ activities in peripheral leukocytes from the four normal adults before biotin administration were within the control ranges determined from studies with 10 additional normal adults shown in Figure 2. Increases in PCC and $\beta \mathrm{MCC}$ activities in leukocyte extracts from the three individuals taking biotin were demonstrable 2 days after starting biotin. PCC activity plateaued after 7-10 days of biotin supplementation at 2-3 times the mean normal activity, while $\beta$ MCC plateaued at 4-9 times the mean normal value. Biotin supplements were stopped after 12 days, but the activities remained elevated for an additional week. Five to six wk after the biotin was stopped, both enzyme activities were again within the normal range. PCC and $\beta \mathrm{MCC}$ activities in leukocyte extracts from the individual not receiving biotin remained within the normal ranges throughout the course of the study (Fig. 2). The total peripheral white blood cell and differential counts did not vary appreciably throughout the study period (data not shown), indicating that biotin did not alter the number or type of leukocytes in any of the individuals.

\section{EFFECT OF BIOTIN ON CARBOXYLASE ACTIVITY IN CONTROL FIBROBLASTS}

Next, the effect of biotin supplements in culture on PCC and $\beta \mathrm{MCC}$ activities were examined in extracts of normal cultured human fibroblasts. PCC, $\beta$ MCC, and GDH activities were assayed in extracts of the three control lines cultured for 4 days. The results are shown in Figure 3. Slight increases in PCC activity, no more than $11 \%$, were observed in extracts from fibroblasts grown in medium containing FBS at the highest concentrations of biotin. A more striking increase in PCC activity, 70-75\%, was observed in extracts from the cells grown in HS with high concentrations of biotin. Maximal activity was observed in cultures grown in medium with $0.1-1.0 \mu \mathrm{g} / \mathrm{ml}$ biotin added and this increased activity was maintained in cultures grown at higher biotin concentrations. An increase in $\beta M C C$ activity of $120-140 \%$ was observed in fibroblast extracts grown in medium with $\mathrm{HS}$ and biotin, with maximal activity achieved, as observed for PCC activity, with $0.1-$ $1.0 \mu \mathrm{g} / \mathrm{ml}$ of biotin in the medium (Fig. 3). GDH, a mitochondrial, nonbiotin requiring enzyme, was assayed in extracts of normal fibroblasts under identical conditions. GDH activity, as shown in Figure 3, did not change appreciably in cells grown in medium supplemented with biotin.

\section{EFFECT OF GROWTH PHASE AND LENGTH OF INCUBATION ON} BIOTIN STIMULATION OF PCC ACTIVITY IN FIBROBLASTS

Stimulation of carboxylase activity as a function of various stages of cell growth and of the time required for maximal activity was investigated. Fibroblasts were plated densely or sparsely in medium containing $10 \% \mathrm{HS}$ and biotin $(1 \mu \mathrm{g} / \mathrm{ml})$. The densely plated cultures were at stationary growth phase and the sparsely plated cultures were in logarithmic growth phase. The cells were cultured for up to 6 days, harvested, pelleted, and frozen at $-68^{\circ} \mathrm{C}$. The log phase cultures had not yet reached confluence by 6 days as evidenced by the finding that total extract protein in these fibroblasts was $20 \%$ less than that in the confluent fibroblasts at the time of plating. Cell extracts were assayed for PCC activity as shown in Figure 4. The maximal increase in activity, about $75 \%$ was achieved in 2-4 days in both the confluent and log phase fibroblast cultures. GDH activity in fibroblasts grown in biotin ( 1 $\mu \mathrm{g} / \mathrm{ml}$ ) did not show a consistent change when monitored at intervals up to 6 days. 


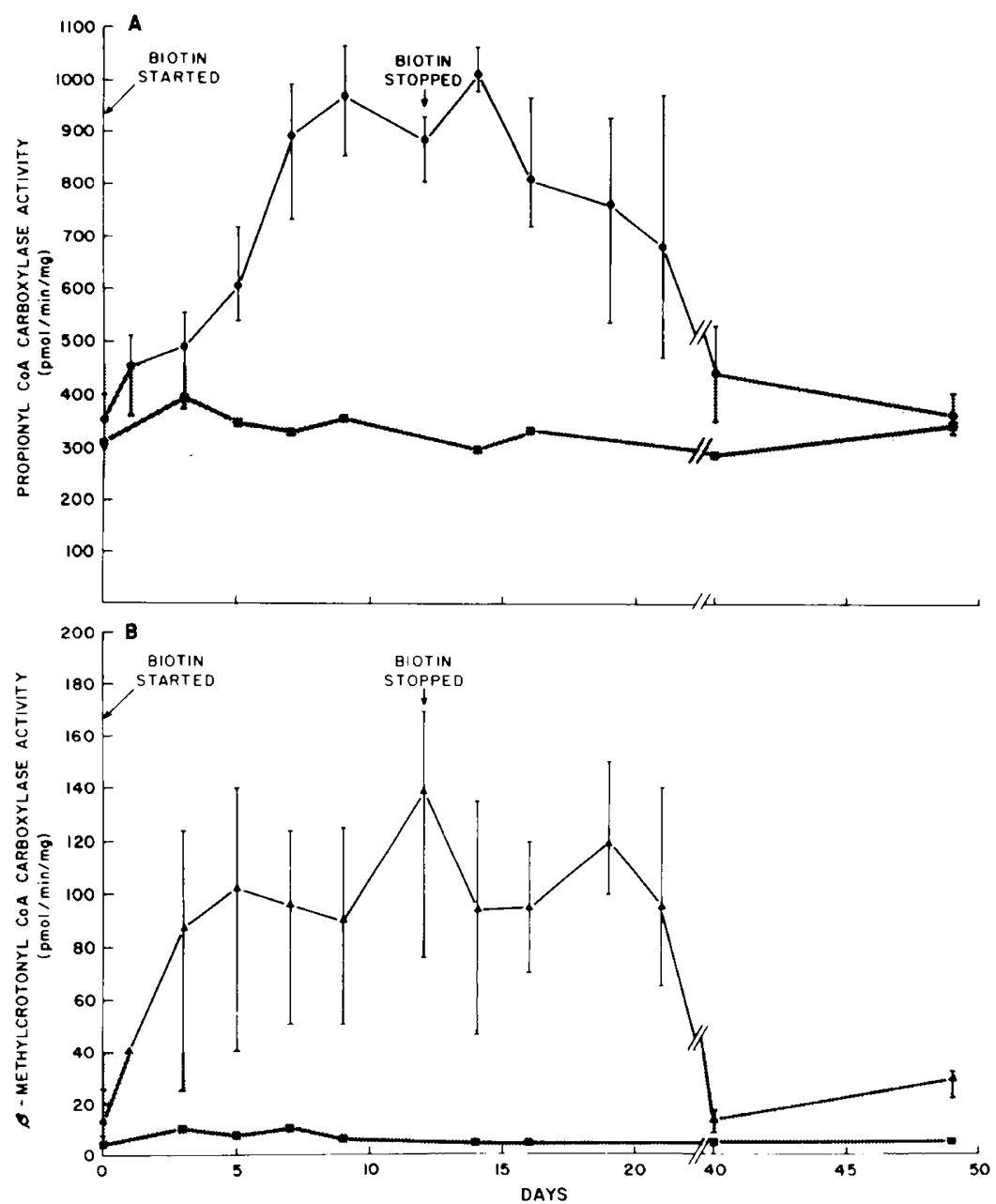

Fig. 2. PCC $(A)$ and $\beta$ MCC $(B)$ activities in peripheral blood leukocytes obtained from four control adults. Three received $20 \mathrm{mg}$ biotin/d $(\bullet$ or $\Delta)$ and one did not receive biotin ( $\square$ ). Activities were measured before biotin was started, while the subjects received biotin (arrow, day 0 ), and after biotin was discontinued (arrow, day 12). The points represent the mean activities of the three controls taking biotin. The vertical bars represent the range of activities of these individuals. The shaded areas represent the normal ranges for PCC and $\beta$ MCC activities for individuals not on vitamin supplements.

\section{EFFECT OF CYCLOHEXIMIDE ON THE STIMULATION OF PCC ACTIVITY IN FIBROBLASTS}

PCC activity was measured in fibroblast extracts from confluent cultures grown in medium containing biotin with and without cycloheximide to determine if the enhanced carboxylase activity was due to de novo protein synthesis. Cultures from three control lines were grown in medium with 10\% HS. Twenty-four hr after the cultures were plated, the medium and serum were discarded, and replaced with fresh medium containing $10 \%$ HS. Biotin (final concentration, $1 \mu \mathrm{g} / \mathrm{ml}$ ) was added to one set of duplicate cultures; cycloheximide $(15 \mu \mathrm{g} / \mathrm{ml})$ was added to a second; to a third set both biotin and cycloheximide were added; and to a fourth set neither biotin nor cycloheximide was added. Cells were then harvested after 4 days of incubation. Identical results were obtained when cycloheximide was added immediately before harvesting to those cultures grown in its absence. Fibroblast viability was $96-100 \%$ in all cultures. The total protein of the harvested pellet was similar, within $4 \%$, in all cultures. The results of three separate experiments, each using cells from a different control line are shown in Table 1. PCC activity in extracts from cultures grown in medium with supplemental biotin was increased above the activity in extracts from cells grown in medium without biotin. A similar percent increase in PCC activity was observed in cultures grown in medium with biotin and cycloheximide compared to cultures grown only in medium with cycloheximide. These data demonstrated that, although cycloheximide decreased PCC activ- ity, this decrease was independent of the biotin content in the medium. Thus, the increased activity in the cultures grown in medium with biotin was not due to de novo protein synthesis.

\section{DISCUSSION}

Although biotin metabolism has been investigated extensively in prokaryotic cells (7), there have been only a few studies in mammalian tissues and these, for the most part, used biotindeficient animals (16). Carboxylase activities in tissues from biotin-deficient animals were reduced significantly $(1,5,19)$, but returned to normal or even above normal values after biotin was returned to the animal's diet $(1,5,19)$. When normal animals received large quantities of oral biotin, their liver pyruvate carboxylase or acetyl $\mathrm{CoA}$ carboxylase activities increased above the normal range $(1,5)$.

The present study demonstrates that $10 \mathrm{mg}$ of oral biotin given twice a day to normal adults (2000 times the minimum daily requirement) was capable of stimulating both PCC and $\beta \mathrm{MCC}$ activity in their peripheral blood leukocytes. Increases in carboxylase activity of leukocytes were detectable within one day of biotin administration; maximum increases, however, were not achieved until 5-10 days. This delay is most certainly not due to the time required for the gastrointestinal absorption of biotin because Horsburgh and Gompertz (10) have shown that a single dose of $10 \mathrm{mg}$ biotin raises the plasma biotin 50 -fold $1 \mathrm{hr}$ after 


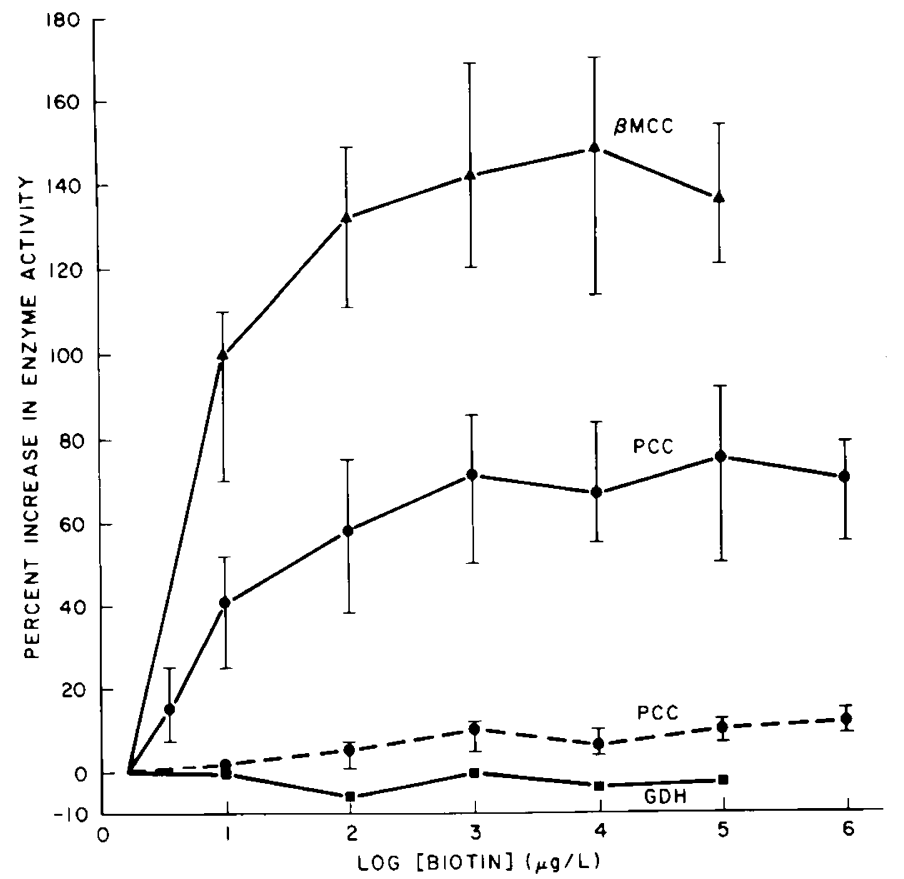

Fig. 3. The percent increase in $\operatorname{PCC}(\boldsymbol{O}), \beta \mathrm{MCC}(\boldsymbol{\Delta})$ and $\mathrm{GDH}(\boldsymbol{\square})$ activities in extracts of normal cultured fibroblasts incubated in medium containing $10 \%$ FBS (----) or $10 \%$ HS (-) with various concentrations of biotin added to the cultures. The percent increases in PCC and $\beta \mathrm{MCC}$ activities represent the mean of three different normal fibroblast lines. Vertical bars denote the ranges of values from these three lines. The percent increases in GDH activity were obtained from a single normal fibroblast line

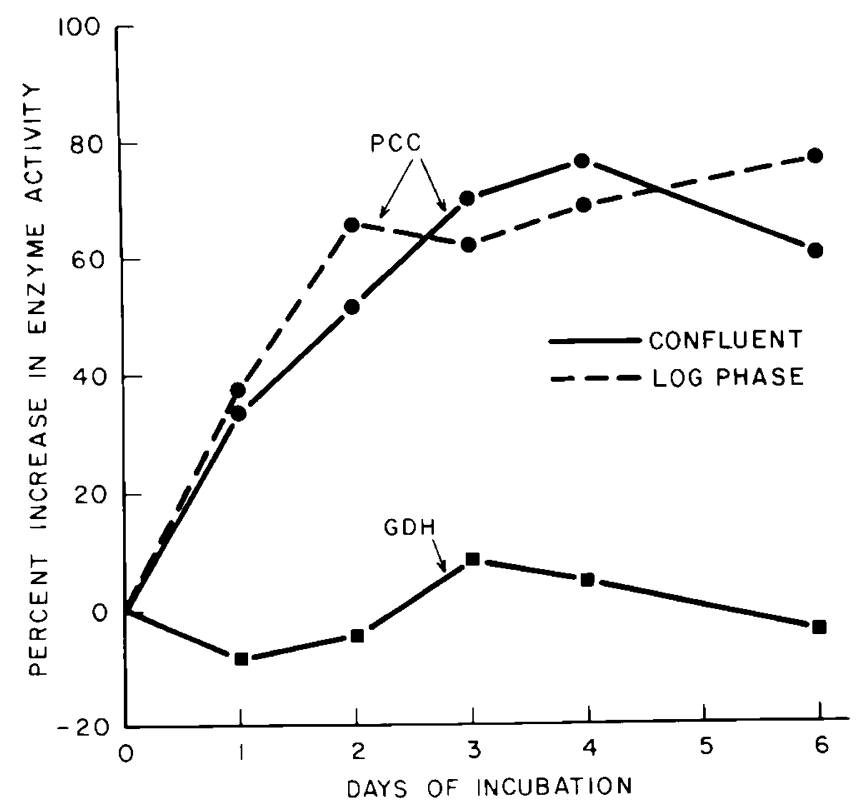

Fig. 4. The percent increase in PCC activity $(O)$ in extracts from normal fibroblast cultures incubated in stationary $(-)$ and logarithmic (-..-) growth phase at various days of incubation in medium containing $10 \% \mathrm{HS}$ and $1 \mu \mathrm{g} / \mathrm{ml}$ biotin. The percent increase in GDH activities ( $\square$ ) in extracts from the same fibroblast cultures are also shown.

ingestion. After $8 \mathrm{hr}$, the plasma biotin concentration was still 10 fold above preload values. Several wk were then required after the withdrawal of biotin before the elevated activities returned to normal. The reason(s) for the delay in initiation and termination of the observed biotin effect in this study is not readily apparent.
Table 1. Effect of cycloheximide on biotin stimulation of PCC activity in control fibroblasts ${ }^{1}$

\begin{tabular}{lccc} 
& Cycloheximide & + Cycloheximide & $\begin{array}{c}\text { Percent de- } \\
\text { crease }\end{array}$ \\
\hline - Biotin & $796 \pm 20^{2}$ & $662 \pm 79$ & $18 \pm 8$ \\
+ Biotin & $1123 \pm 73$ & $936 \pm 108$ & $17 \pm 4$ \\
Percent increase & $41 \pm 9$ & $42 \pm 5$ & \\
\hline
\end{tabular}

' Nearly confluent monolayers of three different control lines were cultured with or without biotin $(1 \mu \mathrm{g} / \mathrm{ml})$ and with or without cycloheximide $(15 \mu \mathrm{g} / \mathrm{ml})$ for $24 \mathrm{hr}$ before measurement of PCC activity in cell extracts. Identical results were obtained when cycloheximide was added, immediately before harvesting, to those cultures grown in its absence. PCC activity is expressed as pmole $/ \mathrm{min} / \mathrm{mg}$ protein.

${ }^{2}$ All values represent mean \pm SD of duplicate observations on the three cell lines.

PCC and $\beta \mathrm{MCC}$ activities were increased in fibroblast extracts from cultures grown in medium containing high concentrations of biotin when compared to the activities in extracts from cultures grown in medium where the only source of biotin was the added serum. This effect was much more pronounced in medium containing human serum than in that containing an equal concentration of fetal bovine serum. No explanation for this difference could be found. Perhaps it reflects the presence of a specific component in human serum which modulates cellular biotin transport, processing, or both.

These results indicate that the maximum enzyme stimulation in normal fibroblast extracts was achieved at $0.1-1 \mu \mathrm{g} / \mathrm{ml}$ of biotin in the medium, a 5- to 50-fold increase in biotin concentration above that in serum. At higher concentrations of biotin, this maximum activity was maintained. If it is assumed that increased carboxylase activity reflects the intracellular biotin concentration, then these results compare well with those of biotin transport experiments in yeast and bacteria (7), in which small increases in the concentration of biotin in the medium resulted in rapid accumulation of intracellular biotin.

The observed stimulation of enzyme activity appears to be independent of fibroblast growth phase (Fig. 4). Because 2-4 days of incubation with biotin are required for maximum PCC activity, the mechanism of stimulation, as in peripheral blood leukocytes, is not immediate, and may require several metabolic steps.

Although oral biotin fed to biotin-deficient rats resulted in increased protein synthesis of several enzymes, these cycloheximide studies suggest that the increased PCC activity in human fibroblasts grown in high concentrations of biotin is not due to de novo protein synthesis. There are, thus, two possible explanations for the increased carboxylase activity seen in cell cultures. First, preexisting apocarboxylase may be activated by supplemental biotin to yield holocarboxylase. Although most of the carboxylase in freshly isolated mammalian tissues is holocarboxylase (17), the proportion of apoenzyme to holoenzyme in human tissues, and particularly in human fibroblast cultures, in which the only source of biotin is the added serum, is not known. Secondly, Landman (12) demonstrated that biotin is covalently attached to the carboxylases after the protomers have been assembled and suggested that two holocarboxylase forms may exist, an active and inactive form. This activation, as shown previously for PCC and other carboxylases, may be due to direct interaction of affector molecules with holocarboxylase $(6,8,13,17,21,22)$ or to enzymatic modification of holocarboxylase, such as by phosphorylation (14). Biotin, therefore, may act directly, by stabilizing PCC, or indirectly, by interacting with other metabolic pathways, producing substances or stimulating enzymes which activate preexisting inactive holocarboxylase.

The present study represents a preliminary report of the stimulation of carboxylase activity by high concentrations of biotin in human cells both in vivo and in culture. The results suggest that such an experimental model may be useful in the investigation of cellular biotin metabolism in man. 


\section{REFERENCES AND NOTES}

1. Atwal, A. S., Robblee, A. R., and Milligan, L. P.: Effects of dietary biotin on liver pyruvate carboxylase and ${ }^{32} \mathrm{P}$ incorporation into nucleic acids in livers of chicks. J. Nutr., 101: 1555 (1971)

2. Barnes, N. D., Hull, D., Balgabin, L., and Gompertz, D.: Biotin-responsive propionic acidemia. Lancet, $2: 244(1970)$

3. Bartlett, K., and Gompertz, D.: Combined carboxylase defect: Biotin-responsiveness in cultured fibroblasts. Lancet, 2: 804 (1976).

4. Beaufay, H., Bendall, D. S., Baudhuin, P., and de Duve, C.: Tissue fractionation studies. 12. Intracellular distribution of some dehydrogenases, alkaline deoxyribonuclease, and iron in rat-liver tissue. Biochem. J., 73: 623 (1959).

5. Chiang, G. S., and Mistry, S. P.: Activities of pyruvate carboxylase and propionyl $\mathrm{CoA}$ carboxylase in rat tissues during biotin deficiency and restoration of the activities after biotin administration. Proc. Soc. Exp. Biol. Med.. 146: 21 (1974).

6. Edwards, J. B., and Keech, D. B.: Activation of pig heart propionyl-CoA carboxylase by potassium ions. Biochim. Biophys. Acta, 159: 167 (1968).

7. Eisenberg, M. A.: Biotin: biogenesis, transport, and their regulation. Adv. Enzymol. 38: 317 (1973).

8. Giorgio, A. J., and Plaut, G. W. E.: The effect of unvalent cations on activities catalyzed by bovine-liver propionyl-CoA carboxylase. Biochim. Biophys. Acta, 139: 487 (1967).

9. Hillman, R. E., Keating, J. P., and Williams, J. C.: Biotin-responsive propionic acidemia presenting as the rumination syndrome. J. Pediatr. 92. 439 (1978)

10. Horsburgh, T., and Gompertz, D.: A protein-binding assay for measurement of biotin in physiological fluids. Clin. Chim. Acta, 82: 215 (1978).

11. Hsia, Y. E., and Scully, K. J.: Propionic acidemia: diagnosis by enzyme assay in frozen leukocytes. J. Pediatr., 83: 625 (1973).

12. Landman, A. D.: Activation of biotin-enzymes: A possible biochemical rationale. Life Sci., 19: 1377 (1976)

13. Lane. D. M., Moss, J., and Polakos, S. E.: Acetyl coenzyme A carboxylase. Curr. Top. Cell. Regul., 8: 139 (1974)

14. Lee, K.-H., and Kim. K.-H.: Regulation of rat liver acetyl coenzyme A carboxylase. Evidence for interconversion between active and inactive forms of enxyme by phosphorylation and dephosphorylation. J. Biol. Chem., 252: 1748 (1977).

15. Lowry, O. H., Rosebrough, N. J., Farr, A. L., and Randall, R. J.: Protein measurement with the folin phenol reagent. J. Biol. Chem., 193: 265 (1951)

16. Mistry, S. P., and Dakshinamurti, K.: Biochemistry of biotin. Vit. Horm., 22: 1 (1964).
17. Moss, J., and Lane, M. D.: The biotin-dependent enzymes. Adv. Enzymol., 35: 321 (1971).

18. Phillips, H. J.: In: P. F. Kruse and M. K. Patterson: Tissue Culture: Methods and Applications, pp 406-407 (Academic Press, New York, 1973).

19. Poston, H. A., and McCartney, T. H.: Effect of dietary biotin and lipid on growth stamina, lipid metabolism and biotin-containing enzymes in brook trout (Sahelinus fontinalis). J. Nutr., 104: 315 (1974).

20. Scriver, C. R., and Rosenberg. L. E.: In: Amino Acid Metabolism and Its Disorders (W. B. Saunders, Philadelphia, 1973).

21. Scrutton, M. C., and Utter, M. F.: Pyruvate carboxylase. IX. Some properties of the activation by certain acyl derivatives of coenzyme A. J. Biol. Chem., 242: 1723 (1967).

22. Scrutton, M. C., and White, M. D.: Purification and properties of human liver pyruvate carboxylase. Biochem. Med., 9: 271 (1974).

23. Weyler, W., Sweetman, L., Maggio, D. C., and Nyhan, W. L. Deficiency of propionyl-CoA carboxylase and $\beta$-methylcrotonyl-CoA carboxylase in a patient with methylcrotonylglycinuria. Clin. Chim. Acta, 76: 321 (1977).

24. Wolf. B., Hsia, Y. E., and Rosenberg, L. E.: Biochemical differences between mutant propionyl CoA carboxylases from two complementation groups. Am. J. Human Genet., 30: 455 (1978).

25. Wolf, B., and Rosenberg, L. E.: Heterozygote expression in propionyl CoA carboxylase deficiency: Differences between major complementation groups. J. Clin. Invest., 62: 931 (1978).

26. Youngdahl-Turner, P.. Mellman, I. S., Allen, R. H., and Rosenberg, L. E. Protein mediated vitamin uptake: Absorptive endocytosis of the transcobalamin II-cobalamin complex by cultured human fibroblasts. Exp. Cell Res.. 118: 127 (1979)

27. The authors thank Dr. Y. E. Hsia for his helpful discussions and Miss M. Feldman and Mrs. L. Herold for their secretarial assistance.

28. This research was supported by a research grant from the National Institutes of Health (AM 12579). Dr. Barry Wolf was a recipient of a traineeship from National Institutes of Health grant GM 00077.

29. The present address of Dr. Barry Wolf is: Departments of Human Genetics and Pediatrics, Medical College of Virginia, Richmond, Virginia 23298, USA.

30. Requests for reprints should be addressed to: Dr. Leon E. Rosenberg, Department of Human Genetics, Yale University School of Medicine, 333 Cedar Street, New Haven, Connecticut 06510, USA.

31. Received for publication July 12, 1978 .

32. Accepted for publication October 24, 1978. 